\begin{tabular}{|l|l|l|l|}
\hline Eiszeitalter u. Gegenwart & Band 26 & Seite 212-217 & Öbringen/Württ. 1975 \\
\hline
\end{tabular}

\title{
Permafrost am Pico de Orizaba/Mexiko
}

\author{
Von Klaus Heine, Bonn ${ }^{1}$ )
}

Mit 5 Abbildungen

$\mathrm{Zusammenf}$ assung. Permafrost tritt in Mexiko (ca. $19^{\circ} \mathrm{N}$ ) am Pico de Orizaba in Höhen zwischen 5000 und $4600 \mathrm{~m}$ auf. Der Permafrost ist fossil. Verschiedene Bodeneistypen können unterschieden werden. Die Permafrostvorkommen unterhalb der Schneegrenze (ca. $5000 \mathrm{~m}$ ) zeigen, daß Schneegrenze und Untergrenze des Permafrostes in Mexiko nicht mit dem von Baranov für die Tropen angegebenen Verlauf übereinstimmen.

$\mathrm{S} \mathrm{u} \mathrm{m} \mathrm{ma} \mathrm{ry.} \mathrm{In} \mathrm{Mexico} \mathrm{(ca.} 19^{\circ} \mathrm{N}$ ) permafrost is found on Pico de Orizaba volcano between 5000 and $4600 \mathrm{~m}$ altitude. The permafrost is fossil. Different types of ground ice can be distinguished. The permafrost occurrence beneath the snowline (ca. $5000 \mathrm{~m}$ ) shows that in Mexico snowline and lower altitudinal limit of permafrost do not agree with the pattern as it is given by Baranov for the tropical zone.

\section{Einleitung}

Wenn der Untergrund nahe der Erdoberfläche, unabhängig von seiner Textur, seinem Wassergehalt oder seinen lithologischen Eigenschaften, von Temperaturen unter $0^{\circ} \mathrm{C}$ für mehr als zwei Jahre ohne Unterbrechung gekennzeichnet wird, so darf man diese Bedingungen als Dauerfrostboden oder Permafrost bezeichnen (WASHBurn 1973). Über Permafrostvorkommen aus subtropischen und tropischen Hochgebirgen liegen bisher nur spärliche Beobachtungen vor (Ives 1974). Aus dem mittelamerikanischen Raum sind meines Wissens Dauerfrostböden bisher nicht beschrieben worden. Am Pico de Orizaba fand ich während verschiedener Jahre Permafrostvorkommen, über die im folgenden kurz berichtet werden soll.

\section{Geländebeobachtungen}

Der Pico de Orizaba (ca. $5700 \mathrm{~m}$ ) befindet sich zwischen $19^{\circ} 00^{\prime}-19^{\circ} 05^{\prime} \mathrm{N}$ und $97^{\circ} 20^{\prime}$ bis $97^{\circ} 13^{\prime} \mathrm{W}$ am Abfall der mexikanischen Meseta zur Golfküstenniederung. Rezente Gletscher (LoRenzo 1964; Abb. 1) bedecken Teile des Kraterkegels. Die Spuren älterer, jungquartärer Vergletscherungen wurden 1974 und 1975 zum ersten Mal für den Gipfelbereich systematisch kartiert. Dabei konnten auch die Beobachtungen über Permafrostvorkommen aus früheren Jahren ergänzt werden.

Am Nord- und Nordosthang befinden sich Permafrostinseln in Höhen zwischen 5000 bis $4600 \mathrm{~m}$. Alle Permafrostbildungen liegen im Bereich der Rundhöckerfluren zwischen den rezenten unteren Gletscherenden und den Hauptendmoränen ( $\mathrm{M} \mathrm{V}$ ) aus dem vergangenen Jahrhundert.

1) Danksagung. Der Deutschen Forschungsgemeinschaft danke ich für eine großzügige Reise- und Sachbeihilfe. Herrn Dr. Dieter Kraus (Bonn) bin ich für die Durchsicht des Manuskripts und die Überlassung der Abb. 4 sowie für einige wertvolle Hinweise zu Dank verpflichtet. 


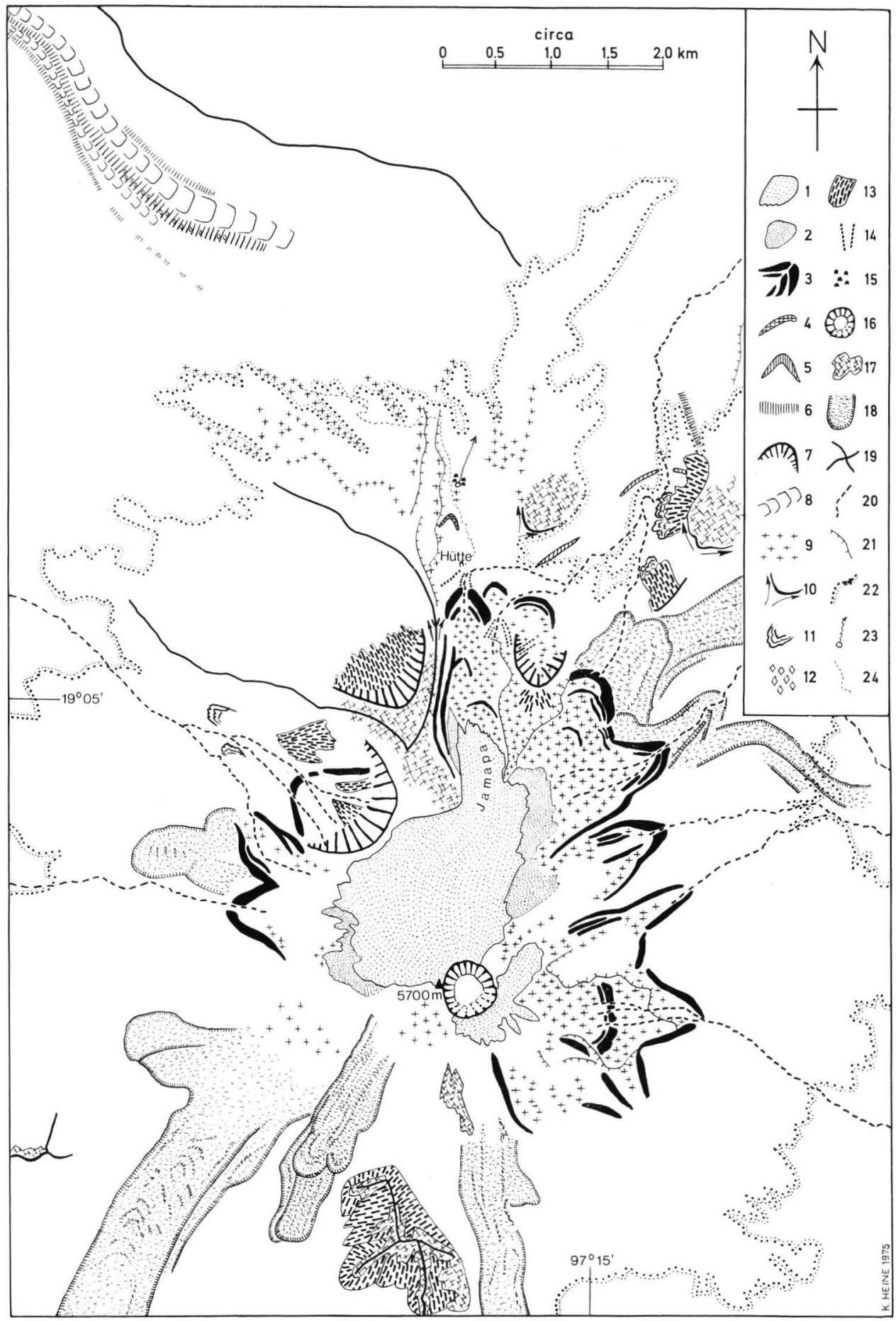

Abb. 1. Glazialmorphologische Skizze des Pico de Orizaba. Es bedeuten: 1 = rezente Gletscher, $2=$ fossiles Eis unter Schutt, $3=\mathrm{M} \mathrm{V-Moränen,} 4=\mathrm{M}$ IV-Moränen, $5=\mathrm{M}$ III 3-Moränen, $6=$ M III 2- und M III 1-Moränen, $7=$ Kar, $8=$ glaziales Trogtal, $9=$ Rundhöcker, $10=$ bei Maximalvergletscherung eisfreie Bergsporne, $11=$ subrezente Blockgletscher, $12=$ geschrammte Blöcke auf Moränen, $13=$ Schutthalden, $14=$ lineare Schuttrutschungen, $15=$ Auffrier-Hügel (Thufer), $16=$ Krater, $17=$ steile Felswände, $18=$ holozäner Lavastrom, $19=$ Grat, $20=$ "Tiefenlinie“ (Tal, Barranca), $21=$ Geländekante, $22=$ obere Waldgrenze (ca. $400 \mathrm{~m}$ Höhe), $23=$ Quelle mit Bach, $24=$ Weg. 
Die Dauerfrostböden zeigen verschiedene Arten des Bodeneises. Legt man die bei Ives (1974) angeführte Klassifikation des Bodeneises für die Unterscheidung zugrunde, so müssen die Bodeneisvorkommen des Pico de Orizaba zum größten Teil der Gruppe VI „Buried Ice" (= begrabenes Eis) zugerechnet werden. Hierbei handelt es sich um fossiles Gletschereis des Gletschervorstoßes aus dem vergangenen Jahrhundert sowie um Permafrost in Moränenmaterial (Abb. 2 u. 3). Fossiles Gletschereis kann Mächtigkeiten von über $7 \mathrm{~m}$ erreichen. Auch die Permafrostvorkommen in M V-Moränen sind - sofern das Moränenmaterial eine entsprechende Mächtigkeit hat - über $10 \mathrm{~m}$ dick. Wie weit der Permafrost in den Untergrund eindringt, konnte nicht ermittelt werden.

Der Dauerfrostboden, der die lockeren Sedimente in den Hohlformen der Rundhöckerfluren zementartig verbacken hat (Abb.3), enthält Bodeneis, das durch Gefrieren von Bodenfeuchtigkeit entstanden ist („Soil Ice“, Gruppe I nach Ives, 1974). Diese Permafrostbildungen erfassen die Grundmoränenschleier, die Mächtigkeiten von wenigen Dezimetern bis zu einigen Metern aufweisen. Ob Temperaturen unter dem Gefrierpunkt auch noch in den anstehenden vulkanischen Gesteinen im Liegenden anzutreffen sind, ist zu vermuten, da Moränenmaterial an anderer Stelle über $10 \mathrm{~m}$ tief vom Permafrost erfaßt wird. Die Untergrenze des Permafrostes wurde nirgends angetroffen.

Der Auftauboden (active layer) über dem Permafrost ist äußerst geringmächtig. Im März 1975 wurden an verschiedenen Tagen am frühen Nachmittag Auftauböden von

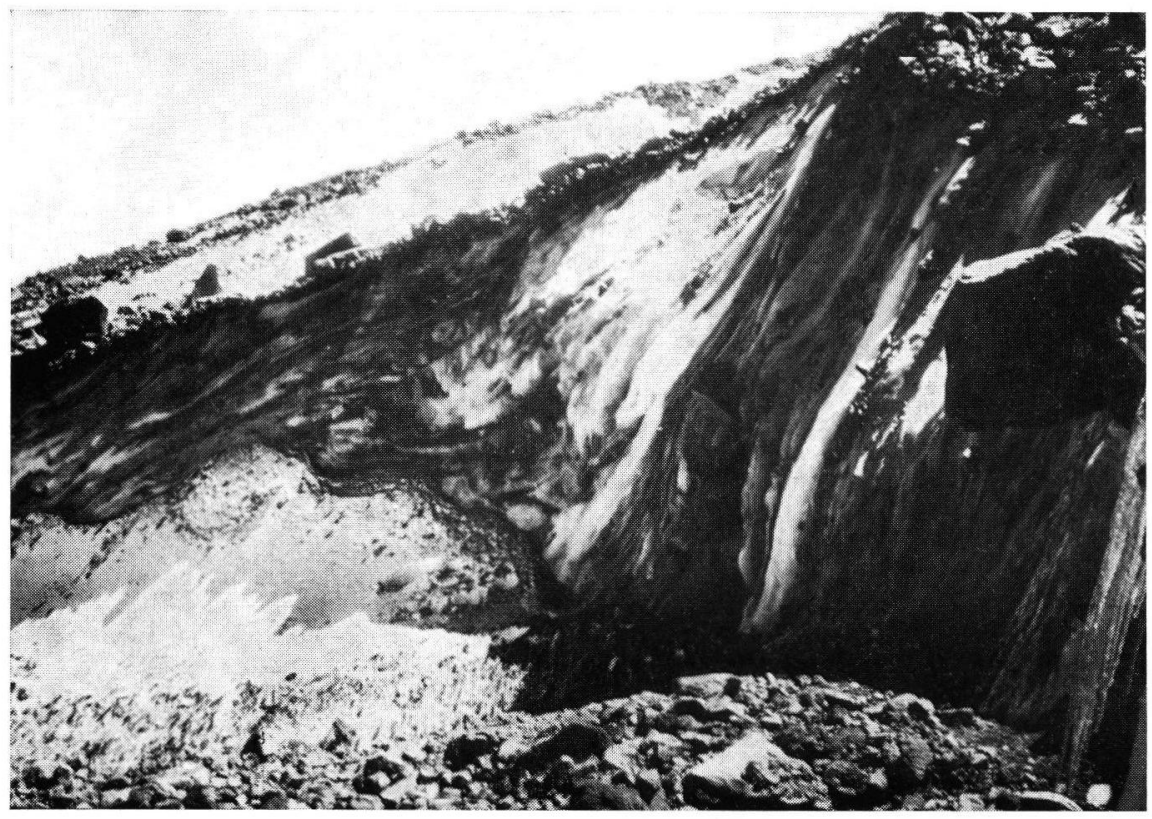

Abb. 2. Eisverbackenes Moränenmaterial, Pico de Orizaba, $4770 \mathrm{~m}$ Höhe. Der Aufschluß befindet sich in einer wallförmigen Ufermoräne. Eis und Moränenmaterial sind zum Teil in Schichten ausgebildet, die an der Innenflanke des Moränenwalles \pm oberflächenparallel (Bildmitte links), zum Moränenkamm hin (rechts) steiler gestellt sein können. Uber dem Permafrost zeigt eine ca. 0,5 m mächtige Schuttlage die Tiefe des "Auftaubodens" (active layer) an. Im Vordergrund ist ausgetautes Moränenmaterial zu erkennen; dahinter liegt im linken Bildteil vor dem Moränenaufschluß eine kleine schneebedeckte (z. T. Büßerschnee) Schutthalde, die den Aufschluß im unteren Teil verdeckt. Aus den Lagerungsverhältnissen des eisverbackenen Moränenmaterials ist auf eine schichtweise Anlagerung des Moränenschutts bei Permafrost zu schließen. Infolge seitlichen Druckes des Gletschers auf das bereits abgelagerte Material erfolgte eine stärkere Schrägstellung im äußeren Teil des Walles (rechts). Die Ufermoräne wurde bis in die Mitte des 19. Jhs. weitergebildet. 


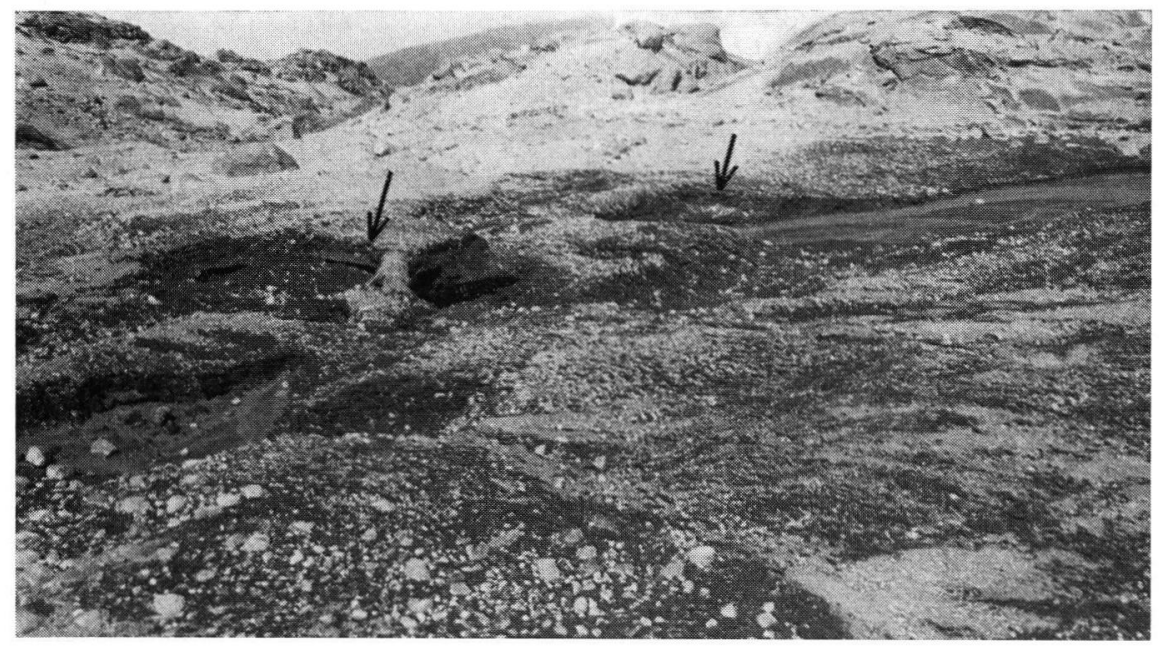

Abb. 3. Permafrost (Pfeile) im Bereich der Rundhöckerflur am Nordhang des Pico de Orizaba in ca. $4850 \mathrm{~m}$ Höhe zwischen den rezenten Gletschern und den Stirnmoränen des 19. Jhs. (M V-Moränen, vgl. Abb. 1). Die Rundhöcker (im Hintergrund) werden aus anstehenden Phänoandesitgesteinen gebildet. Das Bild wurde am frühen Nachmittag aufgenommen. Daher ist der oberflächennahe Schutt aufgetaut und wasserdurchtränkt. Ein Schmelzwasserbach fließt von rechts nach links und hat teilweise die kleinen Aufschlüsse im Dauerfrostboden geschaffen.

10-20 cm Dicke beobachtet. Im Dezember 1971 war über Moränenschutt ein etwa $40 \mathrm{~cm}$ mächtiger Horizont aufgetaut. Die Schuttdecke auf fossilem Gletschereis, die als grober Anhaltspunkt für die maximale Auftautiefe an der Oberfläche angesehen werden kann, beträgt maximal ca. $60 \mathrm{~cm}$ in $4750 \mathrm{~m}$ Höhe.

\section{Diskussion der Ergebnisse}

Die Permafrostvorkommen am Pico de Orizaba sind fossil; darauf deuten alle Beobachtungen hin (fossiles Gletschereis des Vorstoßes des 19. Jhs., starke Schuttanreicherung im Auftauboden gegenüber dem oft sehr stark von Bodeneis bzw. begrabenem Eis durchsetzten Permafrostbereich, etc.). Die Permafrostvorkommen liegen heute deutlich unterhalb der klimatischen Schneegrenze, die bei etwa $5000 \mathrm{~m}$ Höhe angesetzt werden kann. Über einige Klimaparameter am Pico de Orizaba gibt Abb. 4 Auskunft. Geht man von der Erkenntnis aus, daß diskontinuierlicher Permafrost eine Jahresmitteltemperatur von mindestens $-1,0^{\circ} \mathrm{C}$ erfordert (IVEs 1974) und daß die klimatische Schneegrenze in der tropischen Zone i.w.S. mit einer Jahresmitteltemperatur über dem Gefrierpunkt zusammenfällt, so fügen sich die Beobachtungen aus Mexiko nicht in das generalisierte Schema von Baranov (1959, zit. nach Ives 1974) ein; Baranov nimmt an, daß die Untergrenze des Permafrostes in äquatorialen Breiten über der Schneegrenze liegt, in den arktischen Breiten jedoch weit unterhalb der Schneegrenze. Permafrost-Untergrenze und Schneegrenze sollen sich in den Mittelbreiten kreuzen. Demnach müßte die Schneegrenze im tropisch-subtropischen Mexiko unterhalb der Permafrostvorkommen anzutreffen sein, was jedoch nicht zutrifft. Die Gründe dafür sind in verschiedenen Faktoren zu suchen: (1) Die Permafrostvorkommen Mexikos sind fossil; sie werden heute weder aufgebaut noch zerstört. (2) Die Schneegrenze scheint in Mexiko heute weniger durch die Temperatur als 
vielmehr durch die Niederschläge bestimmt zu werden; die Absenkung der Schneegrenze während der M V-Vergletscherung wurde sehr wahrscheinlich zu einem großen Teil durch vermehrte Niederschläge bedingt bei nur geringer Temperaturabsenkung. Darauf deuten auch die Beobachtungen über jungquartäre Schneegrenzdepressionen im Bereich der mexikanischen Vulkangebirge hin (HeINE 1976). (3) Kaltlufteinbrüche („Nortes“) sind während

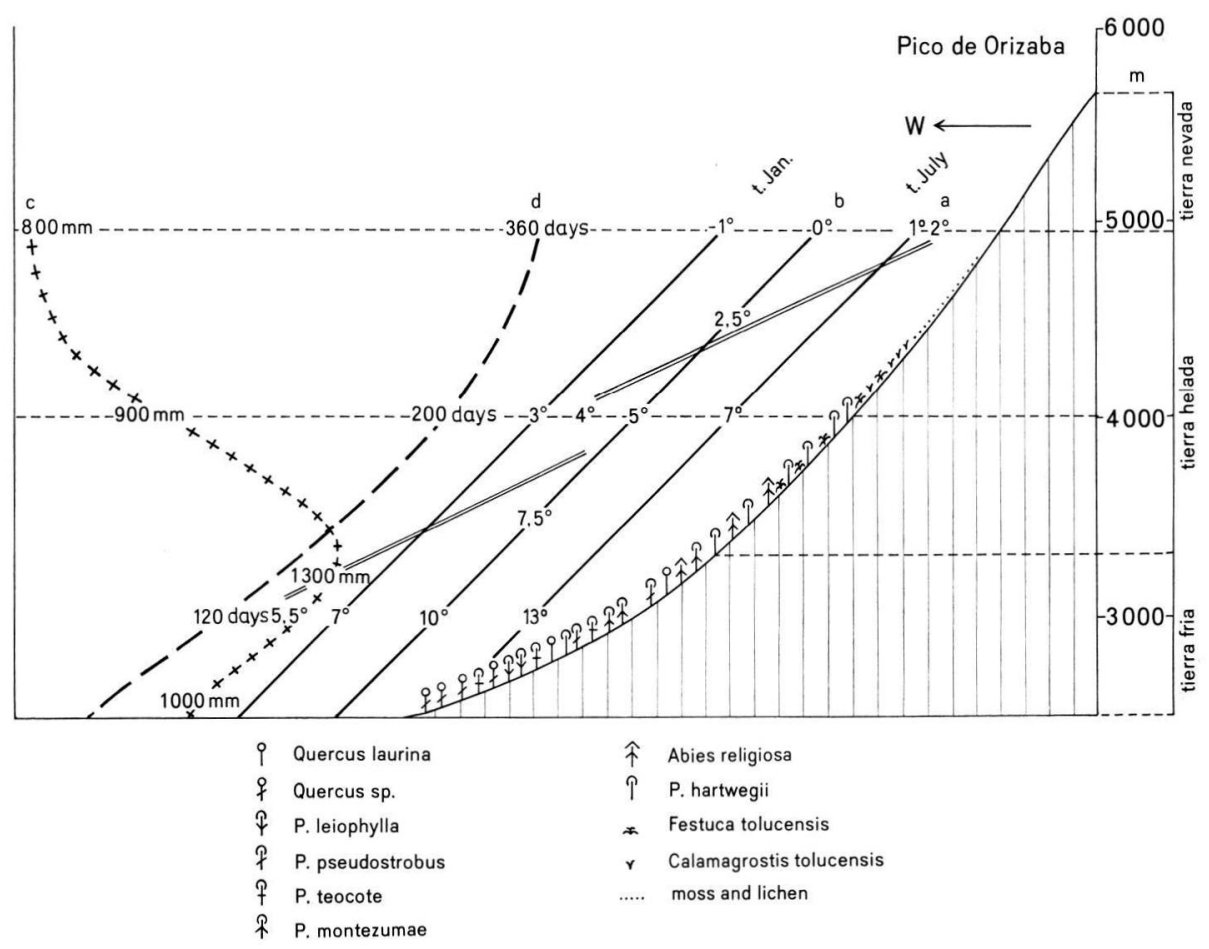

Abb. 4. Approximative Höhenabhängigkeit einiger Klimaparameter am Pico de Orizaba. Es bedeuten: $\mathrm{a}=$ mittlere Jahresschwankung der Temperatur, $\mathrm{b}=$ mittlere Jahrestemperatur, $\mathrm{c}=$ mittlere Niederschläge, $\mathrm{d}=$ mittlere Zahl der Frostwechseltage. Nach Lauer \& Klaus (1975).

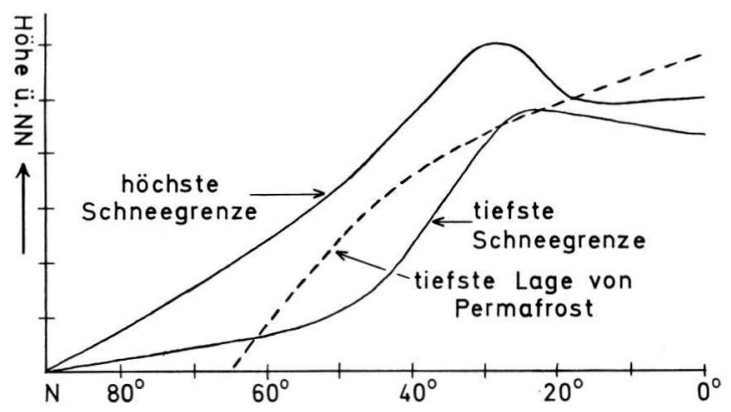

Abb. 5. Schematische Darstellung des Verlaufs der klimatischen Schneegrenze und der montanen Untergrenze von Permafrostvorkommen zwischen Nordpol und Äquator. 
der Monate November bis April oft recht häufig; sie sind als außertropisches Klimaelement anzusehen. Während der Kaltlufteinbrüche tritt in ca. $5800 \mathrm{~m}$ Höhe $(500 \mathrm{mb})$ im Mittel eine Temperaturabsenkung von $2-3^{\circ} \mathrm{C}$ auf.

Die Beziehungen zwischen Schneegrenze einerseits und montaner Untergrenze von Permafrostinseln andererseits müssen daher nicht - wie es BARANOV (1959) annimmt als zwei sich nur einmal im Bereich der Mittelbreiten kreuzende Linien angesehen werden, sondern als Ausdruck von zwei verschiedenen Erscheinungen, die sehr unterschiedlich auf die Klimaelemente Niederschlag und Temperatur reagieren (Abb. 5). Stark generalisierend darf man vielleicht sagen, daß die Untergrenze der Permafrostvorkommen auch im tropisch-subtropischen Bereich in erster Linie von den Temperaturverhältnissen bestimmt wird; die klimatische Schneegrenze dagegen - besonders in den trockenen Tropen und Subtropen - ist sehr stark niederschlagsabhängig.

\section{Schriftenverzeichnis}

Baranov, I. Y.: Geographical distribution of seasonally frozen ground and permafrost (transl. A. Nurklik). - In: Priciples of Geocryology, Part I, Chap. VII, 193-219, NRC Tech. Transl., 1121, Nat. Res. Council, Ottawa 1959.

Heine, K.: Schneegrenzdepressionen, Klimaentwicklung, Bodenerosion und Mensch im zentralmexikanischen Hochland im jüngeren Pleistozän und Holozän. -Z. Geomorph. Suppl.Bd. 24, Stuttgart 1976, im Druck.

Ives, J. D.: Permafrost. - In: Arctic and Alpine Environments, ed. Ives \& Barry, Chap. 4, Sect. A, 159-194, London 1974.

Lauer, W. \& Klaus, D.: Ecological Investigations on the Timberline of Pico de Orizaba/Mexico. - Arctic and Alpine Research 7, im Druck, Boulder 1975.

Lorenzo, S. L.: Los glaciares de México. - UNAM, Monografías del Instituto de Geofísica 1, 1-124, Mexiko 1964.

Washburn, A. L.: Periglacial processes and environments. - 1-320, London 1973.

Manuskript eingeg. 20. 12. 1975.

Anschrift des Verf.: Professor Dr. Klaus Heine, Geogr. Inst. d. Univ. Bonn, Franziskanerstr. 2, D-5300 Bonn 1. 